\title{
The gas-air mixing systems for the industrial gas engine fuelled with low quality gas
}

\begin{abstract}
Modifications of standard fuel system for an industrial gas engine and an analysis of the engine operation on low quality fuel have been described in this paper. The implemented fuel system modifications aim to overcome the challenges which arise when standard fuel gas is replaced by low quality one. Analysis focuses on stability of mixture formation esp. on variations in gas distribution for each cylinder of the engine. The results and discussion include also emissions optimization impact on efficiency as well as the efficiency and emissions level dependency on fuel type.
\end{abstract}

Key words: industrial gas engine, low quality fuels, genset efficiency, emissions

\section{Introduction}

As power consumption globally increases and fossil fuels consumption is scheduled to be reduced, there is a need to look for new fuels for power generation. Renewable fuels are getting more and more attention but the availability is still low and price is high. The worldwide power generation industry turns its eyes towards wastes and the methods for their effective use for power generation.

The industrial waste and biowaste can be turned into gas or liquid fuel. The technologies are already well known and in commercial use for many years. The main challenge is low fuel quality comparing to conventional fossil fuels. Thus, their application using conventional technologies is limited mainly to boiler combined with steam turbine. Such solution is, unfortunately, expensive and inefficient. The industrial gas engine can be considered as interesting alternative, but some challenges need to be solved.

The standard fuel system of the industrial gas engine is designed and optimized for high quality fuel, such as natural gas or biogas. Moreover, the industrial engines require very precise control of mixture composition, to ensure stable operation at desired load profile. Due to this, the gas fuel system needs to be fast in response and should provide proper cylinder balancing.

The important factor is the engine operation optimization which needs to balance two contradicting targets - low emissions and high efficiency. Typical approach is to reach required level of emissions, as an obligation, and the efficiency is a result. However, for low quality waste fuels efficiency is not as important as for good quality ones mainly due to much lower fuel cost. Nevertheless, available fuel energy should have always been used in the most effective way. The engine controls should be set for fuel properties as different fuel type or composition may influence the efficiency and emissions.

\section{Medium pressure gas mixer}

The technology of medium pressure gas mixers is an attractive compromise between low pressure and high pressure system (employing direct gas injection). It provides relatively high safety due to low volume of combustible mixture in the intake manifold when compared with low pressure system. At the same time it doesn't require enor- mous amount of energy for gas compression as in Gas Diesel technology.

There are however, challenges to be overcome. The fuel needs to well mixed with air before it is directed to individual cylinders. Since the fuel dosing system in that concept is located after a turbocharger, which is usually close to the intake manifold the system should employ features enhancing mixture process.

In this study we present current status of a work-inprogress development project of a medium pressure fuel system. So far two different concepts of fuel supply system were tested. The two designs were evaluated in terms of air excess ratio distribution between the engine cylinders.

The fuel system was aimed to effectively work on extremely low calorific gases such as synthesis gas as well as to provide fuel flexibility and work on biogas and natural gas.

The experiments were performed on a model intake system to simulate engine conditions. The tests were done for three gases and the flow conditions were properly adjusted for natural gas, biogas and synthesis gas to ensure required mixture composition for each fuel. Both fuel system concepts were providing the gas just after the charge air cooler in a single point in the center of the intake duct. The only difference between the two fuel system concepts was an additional static mixing element referred to as static mixer, which was only applied in a second design.

The uniformity of gas distribution between the cinders was evaluated by means of normalized equivalence ratio which is an equivalence ratio $(\phi)$ of the gas supplied to a certain cylinder normalized by an average equivalence ratio $\left(\phi_{\text {average }}\right)$ for whole engine. The system performance for natural gas flow settings is shown in Fig. 1.

As shown in Fig. 1 the maximum deviation of normalized equivalence ratio from the average for the base system design (without static mixer) was only $3 \%$. This value was recorded for cylinder number 4 . The additional static mixing element didn't improve the gas distribution. For the design with static mixer the maximum deviation of normalized equivalence ratio has increased up to $8 \%$ (for cylinder number 3).

When the flow settings were changed to simulate flow rates of biogas the results have changed as shown in Fig. 2. 


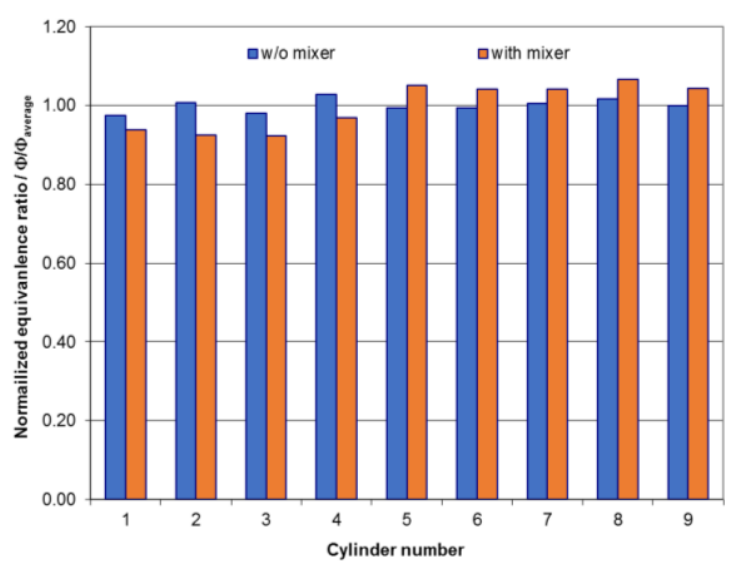

Fig. 1. Comparison of normalized equivalence ratio of two fuel system concepts for natural gas relevant flow conditions

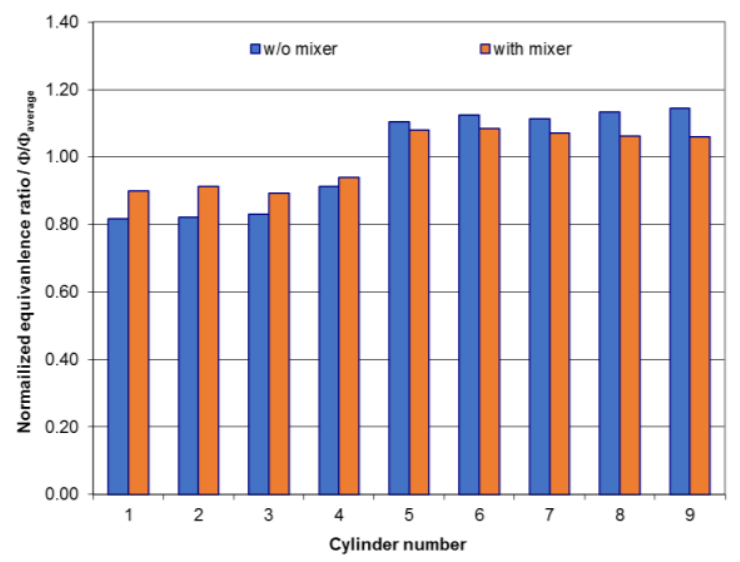

Fig. 2. Comparison of normalized equivalence ratio of two fuel system concepts for biogas relevant flow conditions

The system performance for biogas flow settings has dramatically decreased. The differences in equivalence ratio suggest that most of the gas tends to travel towards one side of the intake receiver. Moreover, in contrary to previous case (natural gas flow settings) in this case the additional static mixing element improved the distribution of the gas.

When the flow settings were changed to simulate mass flow rates of syngas the results have changed again. The results for syngas mass flow settings are presented in Fig. 3.

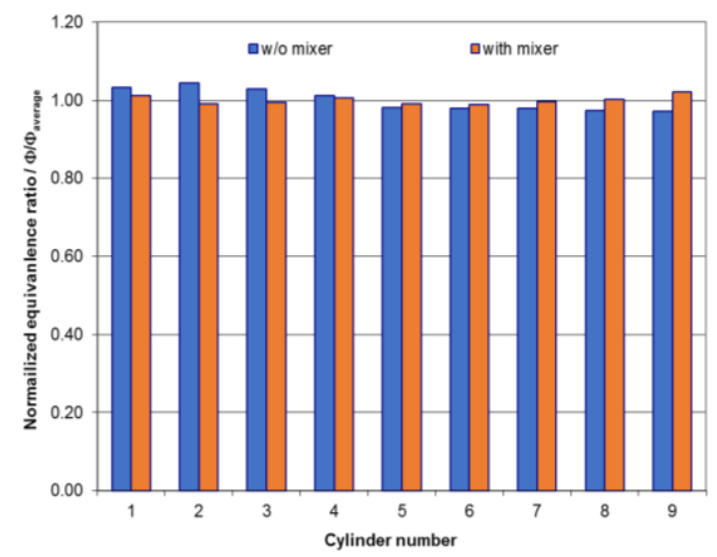

Fig. 3. Comparison of normalized equivalence ratio of two fuel system concepts for syngas relevant flow conditions
For syngas flow settings system performance has been significantly improved. The static mixer has decreased the differences in lambda between the cylinders even more. The system with the additional static mixing element improved the distribution of the gas so the maximum deviation from average was only $2 \%$ for cylinder number 9 .

The obtained results confirmed that proper mixing in medium pressure systems poses a challenge. Although the fuel system design with static mixer provided sufficient performance for syngas flow settings it appeared to be sensitive to the flow conditions, and on other settings the performance has dramatically dropped.

As explained earlier this is a work-in-progress development project and basing on acquired results the next designs will be created.

\section{Low pressure gas mixer}

An advanced gas supply and engine control system HEMUZG can be combined with medium pressure gas fuel injectors or low pressure gas fuel mixer [1, 2, 3]. The system allows continuous control of the air and gas fuel flows and automatic adjustment of each one dependent on engine operation conditions and gas fuel quality. The low pressure gas fuel supply point is located in typical way, i.e. in the engine intake air stream, right after air filter and before inlet to turbocharger compressor.

The location of the gas fuel supply point creates a long distance available for mixing but on the other hand the mixture composition is created globally and cannot be adjusted for each cylinder individually. The final gas fuel delivery to each cylinder was measured indirectly by monitoring the combustion process in each cylinder, for example by measuring the exhaust gas temperature, the knock margin, the spark ignition energy etc. The achieved results for individual cylinders balancing, referred to average, are presented in the following Figs 4 and 5.

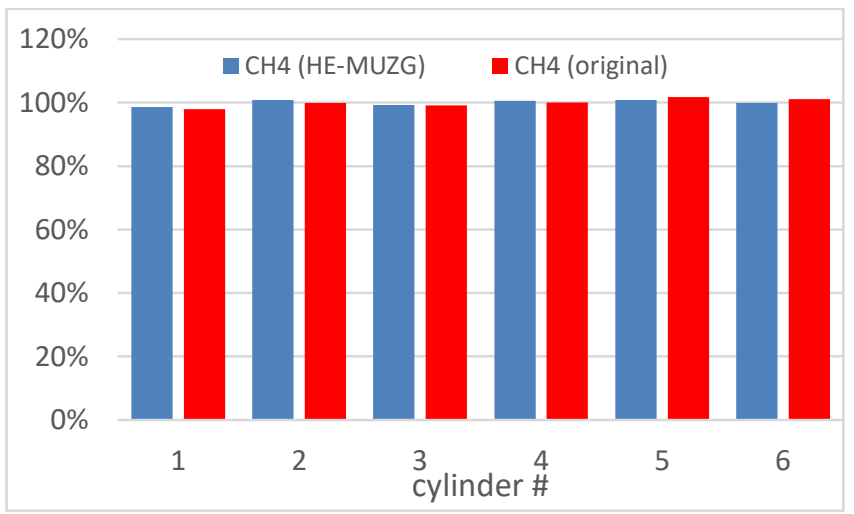

Fig. 4. Individual cylinders balancing comparison based on combustion monitoring for original mixer and HE-MUZG system(balancing referred to average)

The Figure 4 shows that HE-MUZG system provides mixing and cylinder balancing on the same level as standard gas fuel mixer dedicated for methane. The next analysis was done for several fuel types, such as pure propane $\mathrm{C}_{3} \mathrm{H}_{8}$, propene $\mathrm{C}_{3} \mathrm{H}_{6}$ and carbon monoxide $\mathrm{CO}$, biogas (mix of methane $\mathrm{CH}_{4}$ and carbon dioxide $\mathrm{CO}_{2}$ ) and nitrogen-rich natural gas (mix of methane $\mathrm{CH}_{4}$ and nitrogen $\mathrm{N}_{2}$ ). 


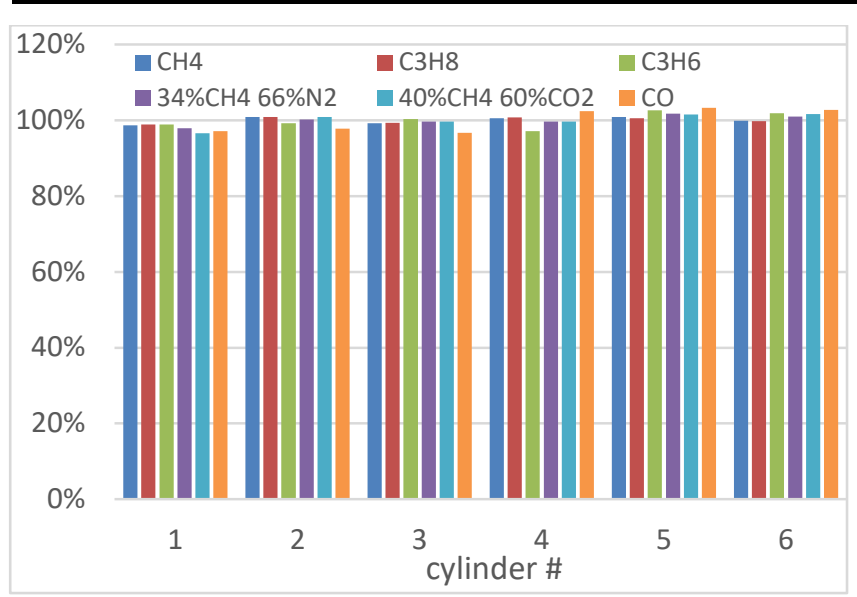

Fig. 5. Individual cylinders balancing comparison for various fuels and HE-MUZG system (balancing referred to average)

The best cylinder balancing was achieved for methane and propane and the worst results were for propene and carbon monoxide. Additional analysis showed that for these two last fuels combustion process is very sensitive and strongly dependent on mixture composition. The carbon monoxide combustion reactions are very slow and even small change in mixture composition can speed up or slow down combustion significantly. On the other hand, the propene is very keen to lead into knock combustion and a small change of mixture stoichiometric coefficient can strongly influence the knock margin. Other observation was that carbon dioxide mixes slightly worse than nitrogen, which is a result of much different molecular weight and density of each gas.

\section{Emissions and efficiency}

The emissions and efficiency test were focused on $\mathrm{NO}_{\mathrm{x}}$ emissions, as this parameter is currently the most challenging to meet. The genset emissions are regulated inde- pendently by each country or region but the most common used is German air quality norm called "TA-Luft", which even if not used directly is very often used as reference. For example: TA-Luft allows $\mathrm{NO}_{\mathrm{x}}$ emission on the level of 500 $\mathrm{mg} / \mathrm{m}^{3}$ of the exhaust gas with $5 \%$ oxygen content and $1 / 2$ TA-Luft allows only half of it [4]. The genset performance tests were carried out for four cases $-\mathrm{NO}_{\mathrm{x}}$ emission according to TA-Luft norm, 1/2TA-Luft, maximum efficiency and the lowest possible emissions enabling stable genset operation. The tests were performed for pure methane, biogas $\left(45 \% \mathrm{CH}_{4}+55 \% \mathrm{CO}_{2}\right)$, methane and propane mix $\left(75 \% \mathrm{CH}_{4}+25 \% \mathrm{C}_{3} \mathrm{H}_{8}\right)$, and carbon; with two load levels: $50 \%$ and $100 \%$ of nominal genset load. The test results are presented into Figures 6 to 9 .

The carbon monoxide tests were very challenging and it was possible to measure emissions and efficiency up to $50 \%$ genset load and only for optimal control settings, i.e. maximum efficiency.

In general the best performance results were achieved for pure methane. The reason is that the engine was originally configured for methane operation and HE-MUZG system influences only controls and fuel supply leaving the engine hardware unchanged.

The well-known relations between efficiency and NOx emissions is clearly visible - the lower emissions the lower efficiency. Recently introduced change of allowed emission level from TA-Luft to $1 / 2$ TA-Luft leads to increase of gas fuel consumption at nominal genset load by $2.7 \%$ for methane $7.9 \%$ for biogas and $13.8 \%$ for methane mixed with propane. Additionally the change from the optimum genset performance to the lowest possible emission results in gas fuel consumption increase by $10.6 \%$ for methane, $12.4 \%$ for biogas and $31.2 \%$ for methane mixed with propane (all for nominal genset load). This reflects in corresponding increase of annual fuel cost.

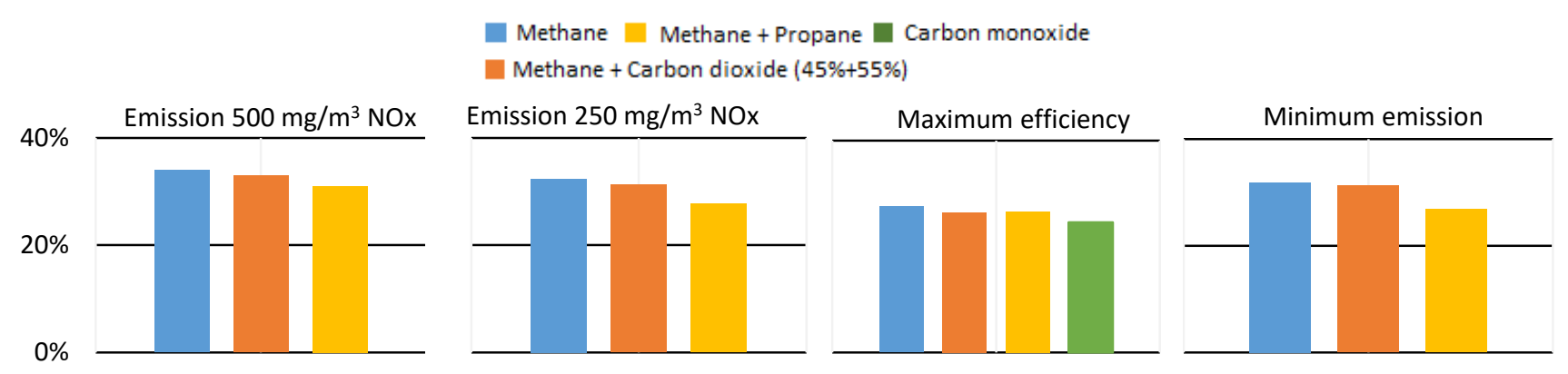

Fig. 6. Genset efficiency at $50 \%$ load for different $\mathrm{NO}_{\mathrm{x}}$ emission settings

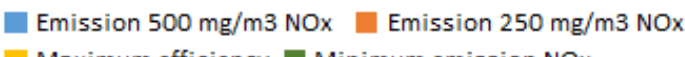

Maximum efficiency Minimum emission NOx
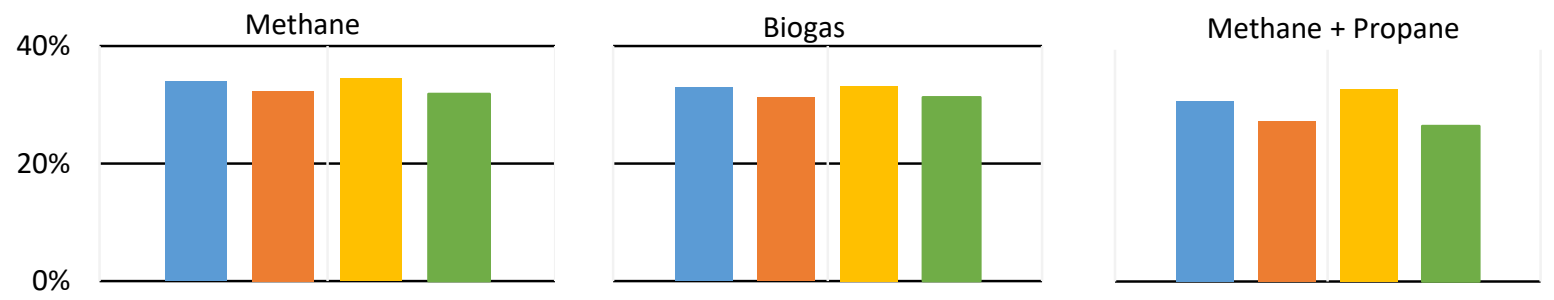

Fig. 7. Genset efficiency at 50\% load for different fuels 


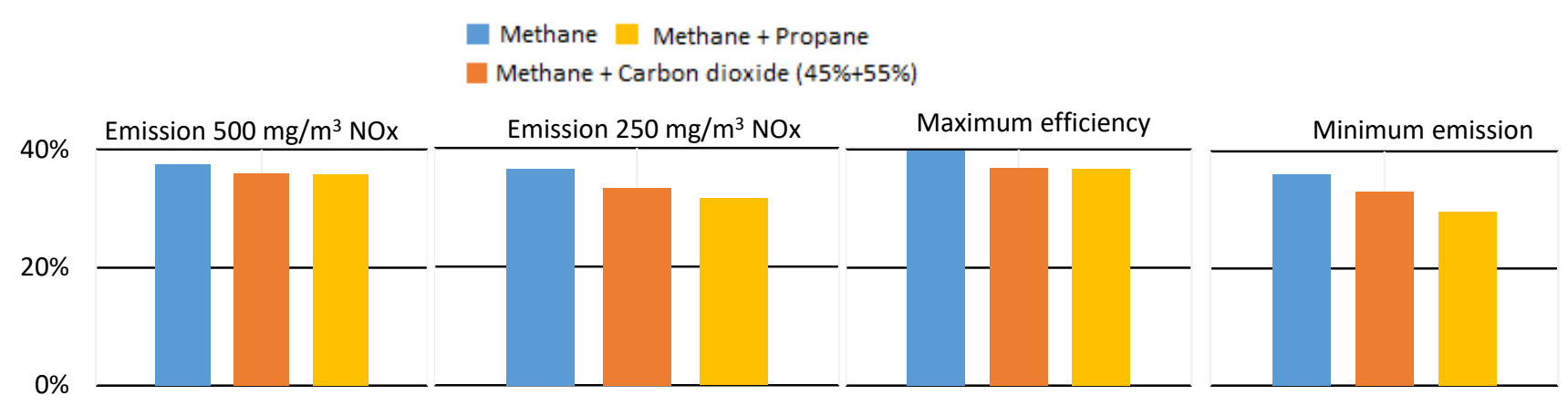

Fig. 8. Genset efficiency at $100 \%$ load for different $\mathrm{NO}_{\mathrm{x}}$ emission settings

Emission $500 \mathrm{mg} / \mathrm{m} 3$ NOx Emission $250 \mathrm{mg} / \mathrm{m} 3 \mathrm{NOx}$

Maximum efficiency $\square$ Minimum emission NOx
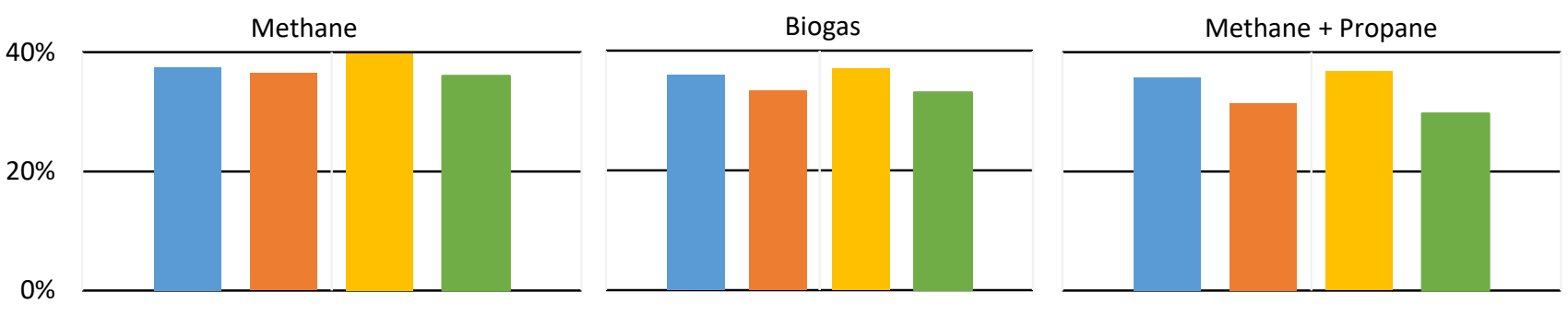

Fig. 9. Genset efficiency at $100 \%$ load for different fuels

\section{Summary and conclusions}

The gas fuel mixing and cylinder balancing results show that much better results are achieved with low-pressure mixer. The main reason is that this solution provides long distance for mixing and also the air and gas passing turbocharger's compressor get additional mixing enforcement. However, it is important to note that medium pressure gas mixer provides much better mixing for syngas than for natural gas. The influence of fuel type is also visible in low pressure gas mixer but the change is not that significant as in medium pressure gas mixer.

The emissions measurements showed two important factors. First of all, the fuel type and related to it combustion process strongly influences the NOx emissions and efficiency. The second factor is that NOx optimisation enforced by legislation can lead to significant increase of power generation sets operational cost due to huge increase in gas fuel consumption.

\section{Bibliography}

[1] BRZEŻAŃSKI, M., MARECZEK, M., SUTKOWSKI, M., SMUGA, W. A 4-stroke spark-ignition engine fuelled with low quality gas. Combustion Engines. 2017, 168(1).

[2] BRZEŻAŃSKI, M., MARECZEK, M., MAREK, W. et al. The executed concept of variable chemical composition fuel gas supply systems for internal combustion engines, Combustion Engines. 2017, 170(3).

\footnotetext{
Marek Sutkowski, DEng. - Advanced Technology Application Manager, Horus-Energia.

e-mail: m.sutkowski@horus-energia.pl
}

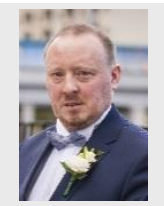

[3] MARECZEK, M., SUTKOWSKI, M., SMUGA, W. Operational tests of an innovative fuel supply system for an industrial 4-stroke gas engines. Scientific Automotive Conference KONMOT 2018. IOP Conference Series. 2018, 421.

[4] PILARSKA, P. Emission measurements of the internal combustion gas engine equipped with an innovative control system. BEng Thesis. Warsaw University of Life Sciences. 2019.

\footnotetext{
Paulina Pilarska, Eng. - Junior Sales Engineer, Horus-Energia.

e-mail: p.pilarska@horus-energia.pl
}

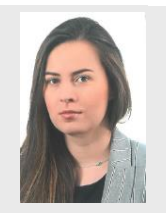

Łukasz Jan Kapusta, DEng. - Faculty of Power and
Aeronautical Engineering, Warsaw University of
Technology.
e-mail: lukasz.kapusta@pw.edu.pl 